\title{
Wide complex tachycardia with changing QRS-axis on loop recorder
}

Luis Quininir ${ }^{1}$, Hariharan Raju ${ }^{2}$, Kim Chan ${ }^{1}$, Chrishan Nalliah $^{3}$, Edward Toal ${ }^{1}$, and Raymond $\mathrm{Sy}^{1}$

${ }^{1}$ Royal Prince Alfred Hospital

${ }^{2}$ Macquarie University

${ }^{3}$ Macquarie University Hospital

September 28, 2020

\section{Wide complex tachycardia with changing QRS-axis on loop recorder}

Short title: QRS-axis changes on loop recorder

\section{Authors:}

Luis Quininir MD a

Hariharan Raju MBChB PhD a, c, d

Kim Chan MBBS, PhD a,b

Chrishan Nalliah MBBS, PhD ${ }^{\mathrm{d}}$

Edward Toal $\mathrm{RN}^{\text {a }}$

Raymond W Sy MBBS, PhD ${ }^{\mathrm{a}, \mathrm{b}, \mathrm{c}}$

Word Count: 756 (inc references)

\section{Affiliations:}

a Department of Cardiology, Royal Prince Alfred Hospital, Sydney, Australia

b Faculty of Medicine and Health, The University of Sydney, Australia,

c Department of Cardiology, Concord Repatriation General Hospital, Sydney, Australia

d Macquarie University, Sydney, Australia

Funding: No external financial support.

Declarations of interest: None.

\section{Address for correspondence:}

A/Prof Raymond Sy, Department of Cardiology, Royal Prince Alfred Hospital, Missenden Rd Camperdown, NSW, Australia

Tel: +612 -9515 8063 Fax: +612 - 95506262 Email: Raymond.sy01@gmail.com 
Keywords: Alternating bundle-branch-block, Implantable loop recorder, Rhythm monitoring, Troubleshooting

\section{Case:}

A 74-year-old female was admitted due an episode of loss of consciousness with a 3-year history of recurrent syncope and previous loop recorder implant (Medtronic Reveal Linq $\mathbf{R})$ ). There was no history of coronary artery disease, structural heart disease, or other cardiovascular implantable electronic devices (CIED). Resting echocardiogram and stress testing were normal. Electrocardiogram (ECG) at baseline showed left bundle branch block (LBBB) (Figure 1), with no history of pre-excitation. She was not on antiarrhythmic medications. Interrogation of the device showed multiple episodes of wide complex tachycardia temporally unrelated to the loss of consciousness, with a changing QRS morphology (Figure 2). What are the differential diagnoses for the arrhythmia?

\section{Commentary}

Careful examination of the loop recorder tracing showed a wide complex tachycardia with mild variability in cycle length (TCL) 280-340 ms, followed by abrupt termination into presumed sinus rhythm. The QRS morphology changed during the tachycardia. The initial QRS morphology (qR), was followed by a transition zone and then a different QRS morphology (QS) (Figure 2). The latter was similar, although not identical to the QRS morphology during sinus rhythm.

Initial differential diagnoses included supraventricular tachycardia (SVT) with alternating bundle branch block (BBB), polymorphic ventricular tachycardia (PMVT), Ventricular tachycardia (VT) with >1 exit site, SVT with bystander pre-excitation, and dual tachycardia with progressive fusion from ventricular tachycardia to SVT with BBB (or vice-versa).

SVT with alternating BBB was excluded because it would not explain the transition zone where fusion was observed. The appearance was also not consistent with PMVT because the initial and terminal component of the tachycardia showed a consistent 'monomorphic' appearance. A single VT with 2 distant exit sites was felt to be extremely unlikely in the absence of structural heart disease. SVT with bystander pre-excitation could not be excluded, but the patient never exhibited pre-excitation on multiple baseline ECGs. Dual tachycardia with progressive fusion transitioning between VT and SVT was possible, but unlikely given the changing QRS morphology was observed repeatedly during multiple arrhythmia episodes, and an abrupt change in cycle length was not observed coincident with the change in QRS morphology. Given that the clinical history was not consistent with a high probability of VT or pre-excitation, we then also considered the possibility of an artefactual cause for the QRS change.

On physical examination and interrogation of ILR, the loop recorder was implanted in the 4th intercostal space in a parasternal position and rotation of the patient from decubitus supine to left lateral position reproduced a change in QRS morphology similar to that observed during tachycardia. (Figure 3)

Hence, the tachycardia was thought to be most likely supraventricular origin, with pseudo-QRS alternans due to change of ILR position.

The Medtronic Reveal Linq stores the recorded bipolar signal, obtained with sensing electrodes built into the ILR shell. Changes in the device position against the heart would lead to changes in 'far field' QRS morphology. To our knowledge, there is at least one other case report of position-related pseudo-alternating $\mathrm{BBB}$ on implantable loop recorder [1].

Positional change in the recording vector should be considered as an important differential diagnosis when apparent QRS morphology changes are observed during interrogation of an ILR and possibly other CIEDs employing a subcutaneous vector for ECG acquisition. A gradual transition in QRS morphology provides a clue and it can be confirmed with ILR recording during dynamic manoeuvres.

\section{REFERENCES}


Perrin T, Kühne M, Novak J. Positional pseudo-alternating bundle-branch-block on implantable loop recorder electrogram. Indian Pacing Electrophysiol J . 2018;18(6):240-241. doi:10.1016/j.ipej.2018.09.001

\section{FIGURES}

Figure legend $: \mathrm{LBBB}=$ left bundle block, $\mathrm{VS}=$ ventricular sense, $\mathrm{TS}=$ tachycardia sense

Figure 1

Baseline ECG: Sinus rhythm with LBBB.

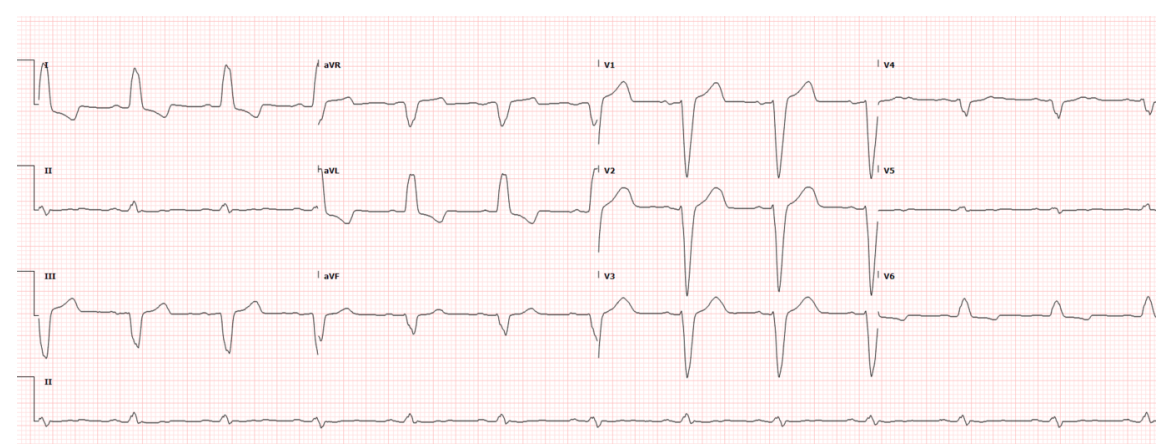

\section{Figure 2}

Implantable loop recorder tracing during tachycardia detection. Speed 25mm/s.

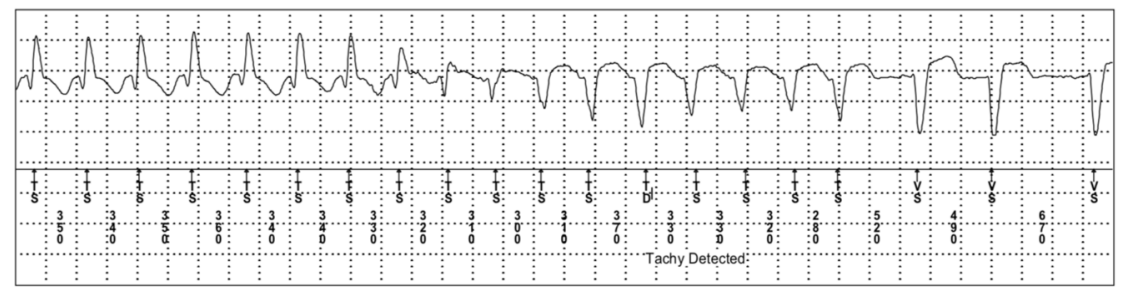

\section{Figure 3}

Implantable loop recorder interrogation during position changes. Speed 25mm/s. 

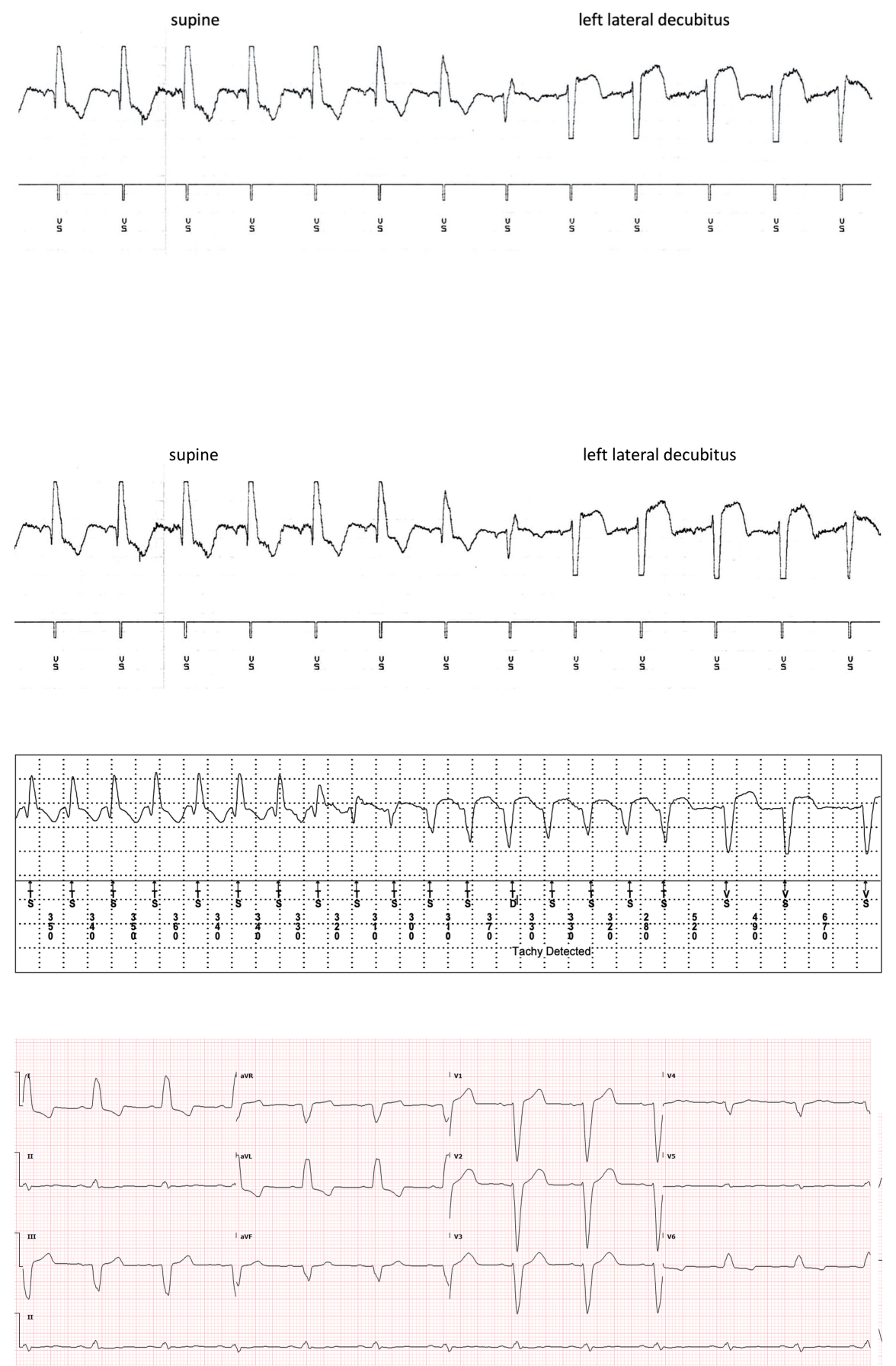